Article

\title{
Prevalence and Correlates of Sexual Risk Behavior among School-Going Adolescents in Four Caribbean Countries
}

\author{
Supa Pengpid ${ }^{1,2}$ (D) and Karl Peltzer ${ }^{3, *(D)}$ \\ 1 ASEAN Institute for Health Development, Mahidol University, Salaya, Phutthamonthon, \\ Nakhon Pathom 73170, Thailand; supa.pen@mahidol.ac.th \\ 2 Department of Research Administration and Development, University of Limpopo, \\ Turfloop 0727, South Africa \\ 3 Department of Psychology, University of the Free State, Bloemfontein 9300, South Africa \\ * Correspondence: kfpeltzer@gmail.com
}

Received: 18 September 2020; Accepted: 19 October 2020; Published: 29 October 2020

\begin{abstract}
This study aimed to assess the prevalence and correlates of sexual risk behaviors among adolescents in the Caribbean. Nationally representative cross-sectional data were analyzed from 9143 adolescents (15 years = median age) that took part in the 2016 Dominican Republic, 2016 Suriname, 2017 Jamaica, and 2017 Trinidad and Tobago Global School-Based Student Health Survey (GSHS). The results indicate that $41.4 \%$ of the students had ever had sex, ranging from $26.4 \%$ in Trinidad and Tobago to $48.1 \%$ in Jamaica. Among the sexually active, $58.8 \%$ had had $\geq 2$ sexual partners; $58.6 \%$ had had an early sexual debut ( $\leq 14$ years); $41.9 \%$ had not used birth control the last time they had sex; $28.4 \%$ had not used a condom the last time they had sex; and, of the whole sample, $31.9 \%$ had engaged in two or more (multiple) sexual risk behaviors, ranging from $16.5 \%$ in Trinidad and Tobago to $40.3 \%$ in Jamaica. In an adjusted logistic regression analysis, substance use (tobacco, alcohol, and cannabis), psychological distress, frequent soft drink intake, participation in physical fighting, school truancy, older age, and male sex were associated with single and/or multiple sexual risk behaviors. A large number of adolescents in the Caribbean reported sexual risk behaviors, emphasizing the need for intervention.
\end{abstract}

Keywords: sexual behavior; adolescents; health risk behavior; Caribbean

\section{Background}

Sexual initiation often occurs during the adolescent period and is associated with unprotected sex and other sexual risk behaviors [1]. Among adolescents in Caribbean countries, various sexual risk behaviors, such as early sexual debut and unprotected sex, have been identified, including its consequences of sexually transmitted infection and adolescent pregnancy [2,3]. The adolescent fertility rate (defined as the number of births per 1000 women aged 15 to 19 years) is 63.8 (per 1000 adolescent women) in the Caribbean (108.7 in the Dominican Republic and 77.3 in Jamaica), which surpassed the global average of 55.7 per 1000 adolescent women [2]. Overall, the Caribbean adult (15-49 years) HIV prevalence was 1.1\%, and one third of new HIV infections in the Caribbean in 2019 were among young people aged 15-24 [4]. About 40\% of boys and 20\% of girls (13-15 years) had initiated sex [2], and of those sexually active, $79 \%$ of boys and $56 \%$ of girls had had an early sexual debut ( $<14$ years) in the Caribbean [2]. Almost one in three boys (31\%) and 10\% of girls (13-15 years of age) had multiple sexual partners [2], and 38\% did not use a condom the last time they had sex [2]. The prevalence of early sexual debut (<15 years) was $26.9 \%$ in six Caribbean countries in 2007-2009 (13-16 years) [5]. In several local studies among adolescents in Jamaica, high sexual risk behavior has been reported-e.g., in a 
subsample of adolescents (15-19 years) in 2008-2009, 32\% of females and 54\% of males had had sexual initiation, and among sexually active girls and boys $12 \%$ and $52 \%$, respectively, had had multiple sexual partners [6]. An adolescent school survey (13-18 years) in Kingston and St Andrew in 2012 showed that $58.6 \%$ reported sexual initiation and $31.8 \%$ had had multiple sexual partners [7]; among sexually active youth (15-24 years) in Jamaica in 2012, 58.8\% reported inconsistent condom use and $44.5 \%$ had had multiple sexual partners [8].

In a review among adolescents in the English-speaking Caribbean, "the quality of the parent-adolescent relationship, the presence of violence, substance abuse or mental health problems in the family, peer relationships, cultural attitudes, and a history of physical and sexual abuse" were associated with several sexual and reproductive health outcomes [9]. Globally, as reviewed in Peltzer and Pengpid [10] (p. 406), "factors associated with sexual risk behavior among adolescents (ever had sex, early sexual debut, no condom use, and no contraceptive use), include, male sex, older age, substance use, psychological distress, school truancy, lack of parental and peer support." In addition, in a multi-country adolescent study, frequent bullying victimization increased the odds of sexual initiation, non-condom use, and multiple sex partners [11]; frequent involvement in physical fighting increased the odds of sexual initiation and multiple sex partners [12]; in American adolescents, frequent soda consumption increased the odds of multiple sexual partners and unprotected sex [13]; and in a study of college students in Zambia, low physical activity increased the odds of any risky sexual behavior [14].

However, there is a lack of recent national data on adolescent sexual risk behavior and its correlates in the Caribbean, such as in Dominican Republic, Jamaica, Suriname, and Trinidad and Tobago. Knowing the occurrence and factors associated with sexual risk behavior and its associated factors among adolescents in Caribbean countries will help in informing intervention strategies targeting the delay of sexual initiation and promoting "safer sex". According to Joint United Nations Programme on HIV/AIDS (UNAIDS), "Safer sex strategies include postponing sexual debut, non-penetrative sex, correct and consistent use of male or female condoms, and reducing the number of sexual partners" [15] (p. 11).

Therefore, this study intended to estimate the prevalence and predictors of sexual risk behaviors among school adolescents in Dominican Republic, Jamaica, Suriname, and Trinidad and Tobago in 2016-2017.

\section{Methods}

\subsection{Sample and Procedures}

Cross-sectional nationally representative survey data from the 2016 Dominican Republic, 2016 Suriname, 2017 Jamaica, and 2017 Trinidad and Tobago GSHS were analyzed [16]. These countries were selected on the basis of all the countries in the Caribbean where recent GSHS data were available. A two-stage cluster sample design was utilized to generate representative data of all students in Forms 1-6 in the Dominican Republic, Jamaica, Suriname, and Trinidad and Tobago [16]. At the first stage, schools were selected with a probability proportional to the enrolment size, and at the second stage classes were randomly selected and all the students in the selected classes were eligible to participate [16]. Data collection was done with a self-administered multichoice format questionnaire translated into the country language under the supervision of trained external survey administrators in the classroom [16]. Students recorded their responses on computer scannable sheets, and student privacy was maintained through anonymous and voluntary participation [16]. More detailed information can be publicly accessed [16]; the overall response rate was 63\% in the Dominican Republic, 60\% in Jamaica, 83\% in Suriname, and 89\% in Trinidad and Tobago [16]. National ethics committees approved the study and written informed consent was obtained from the participating schools, parents, and students [16].

The study context characteristics of the four study countries are described in Table 1. 
Table 1. Study context characteristics.

\begin{tabular}{|c|c|c|c|c|}
\hline Variable & Dominican Republic [17] & Jamaica [18] & Suriname [19] & Trinidad and Tobago [20] \\
\hline Population & $10,499,704$ & $2,808,570$ & 609,569 & $1,208,789$ \\
\hline Urban population & $82.5 \%$ & $56.3 \%$ & $66.1 \%$ & $53.2 \%$ \\
\hline Major ethnic groups & $\begin{array}{c}\text { mixed } 70.4 \% \text { (mestizo/indio } \\
58 \% \text {, mulatto } 12.4 \% \text { ) } \\
\text { black } 15.8 \%\end{array}$ & $\begin{array}{c}\text { black } 92.1 \% \text {, mixed } \\
6.1 \%\end{array}$ & $\begin{array}{c}\text { Hindustani } 27.4 \% \text {, "Maroon" } \\
\text { 21.7\%, Creole } 15.7 \% \text {, } \\
\text { Javanese } 13.7 \%\end{array}$ & $\begin{array}{c}\text { East Indian } 35.4 \%, \\
\text { African descent } 34.2 \% \text {, } \\
\text { mixed/other } 15.3 \%\end{array}$ \\
\hline $\begin{array}{c}\text { Secondary school gross } \\
\text { enrolment ratio, female [21] }\end{array}$ & $85 \%$ & $86 \%$ & $89 \%$ & $88 \%$ \\
\hline $\begin{array}{l}\text { Secondary school gross } \\
\text { enrolment ratio, male [21] }\end{array}$ & $78 \%$ & $85 \%$ & $67 \%$ & $83 \%$ \\
\hline $\begin{array}{l}\text { Study sample: Mean age } \\
\text { (SD), age range }\end{array}$ & $14.9(1.5), 11-18$ years & $\begin{array}{c}15.0(1.3), 11-18 \\
\text { years }\end{array}$ & $14.8(1.7), 11-18$ years & $14.8(1.7), 11-18$ years \\
\hline \multicolumn{5}{|l|}{ School grade } \\
\hline 1st Form & $426(29.4 \%)$ & $41(2.5 \%)$ & $642(31.0 \%)$ & $896(23.3 \%)$ \\
\hline 2nd Form & $394(27.2 \%)$ & $412(25.0 \%)$ & $678(32.7 \%)$ & $667(17.4 \%$ \\
\hline 3rd Form & $317(21.9 \%)$ & $557(33.8 \%)$ & $691(33.3 \%)$ & $851(22.1 \%)$ \\
\hline 4th Form & $216(14.9 \%)$ & $388(23.5 \%)$ & $61(2.9 \%)$ & $767(20.0 \%)$ \\
\hline 5th Form & $94(6.5 \%)$ & $208(12.6 \%)$ & $0(0.0 \%)$ & $462(12.0 \%)$ \\
\hline 6th Form & $0(0.0 \%)$ & $44(2.7 \%)$ & $0(0.0)$ & $200(5.2 \%)$ \\
\hline
\end{tabular}

\subsection{Measures}

The GSHS questionnaire used is shown in Table 2 [16]. The GSHS measure draws on content from the Centers for Disease Control and Prevention (CDC) Youth Risk Behavior Survey, for which the test- and retest reliability has been established [22]. A study examining the test-retest reliability of the GSHS measure among Fijian girls found an "average agreement between test and retest was 77\%, and average Cohen's kappa was 0.47." [23] (p. 181).

Sexual risk behavior was assessed with questions on ever having had sexual intercourse, the age of sexual debut, the number of people having had sexual intercourse within a lifetime, condom use at the last sexual intercourse, and birth control use at the last sexual intercourse. Individual sexual risk behaviors were defined as ever having had sex, early sexual debut ( $\leq 14$ years), having had two or more sexual partners in a lifetime, non-condom use in the last sexual encounter, and non-birth control use in the last sexual encounter. A composite sexual risk behavior measure included having had sex, early sexual debut ( $\leq 14$ years), having had two or more sexual partners in a lifetime, and non-condom use in the last sexual encounter; non-birth control use was excluded due to overlap with non-condom use in the last sexual encounter.

Emotional-contextual factors include factors such as bullying victimization, being in physical fights, hunger, parental tobacco use, passive smoking, school truancy, and psychological distress variables (loneliness, anxiety, no close friends, suicidal ideation, and suicide attempt) that were summed and grouped into $0=0,1=1$ single, and 2-5 $=2$ multiple psychological distress [24].

Health risk behaviors included current tobacco use, current cannabis use, lifetime drunkenness, attendance of physical education, injury, and soft drink consumption.

Protective factor items included peer support and four parental support items (connectedness, supervision, bonding, and respect for privacy). The latter were summed and classified into three groups, 0-1 low, 2 medium, and 3-4 high support [24]. 
Table 2. Questionnaire items.

\begin{tabular}{|c|c|c|}
\hline Indicator & Item & Responses (Coding Scheme) \\
\hline Sex & "What is your sex?" & "Male, Female" \\
\hline Age & "How old are you?" & "11 years old or younger to 18 years old or older" \\
\hline \multicolumn{3}{|c|}{ Sexual behavior } \\
\hline Sexual initiation & "Have you ever had sexual intercourse?" & "Yes, No" (coded yes $=1$, no $=0$ ) \\
\hline Age of sexual initiation & $\begin{array}{l}\text { "How old were you when you had sexual intercourse } \\
\text { for the first time?" }\end{array}$ & $\begin{array}{l}\text { "I have never had sexual intercourse } \\
11 \text { years old or younger to } 18 \text { years old or older" }\end{array}$ \\
\hline Number of sex partners & $\begin{array}{c}\text { "During your life, with how many people have you } \\
\text { had sexual intercourse?" }\end{array}$ & $\begin{array}{c}\text { "I have never had sexual intercourse, } 1 \text { person to } 6 \text { or } \\
\text { more people" }\end{array}$ \\
\hline Condom use & $\begin{array}{c}\text { "The last time you had sexual intercourse, did you or } \\
\text { your partner use a condom?" }\end{array}$ & $\begin{array}{c}\text { "I have never had sexual intercourse, Yes, No, I do } \\
\text { not know" }\end{array}$ \\
\hline Birth control use & $\begin{array}{l}\text { "The last time you had sexual intercourse, did you or } \\
\text { your partner use any method of birth control, such as } \\
\text { withdrawal, rhythm (safe time), birth control pills, or } \\
\text { any other method to prevent pregnancy?" }\end{array}$ & $\begin{array}{l}\text { "I have never had sexual intercourse, Yes, No, I do } \\
\text { not know" }\end{array}$ \\
\hline \multicolumn{3}{|l|}{ Emotional-contextual factors } \\
\hline No close friends & "How many close friends do you have?" & $" 1=0$ to $4=3$ or more $(\operatorname{coded} 1+=0,0=1) "$ \\
\hline Loneliness & $\begin{array}{l}\text { "During the past } 12 \text { months, how often have you felt } \\
\text { lonely?" }\end{array}$ & $" 1=$ never to $5=$ always $(\operatorname{coded} 1-3=0$ and $4-5=1) "$ \\
\hline Worry/Anxiety & $\begin{array}{l}\text { "During the past } 12 \text { months, how often have you } \\
\text { been so worried about something that you could not } \\
\text { sleep at night?" }\end{array}$ & $" 1=$ never to $5=$ a lways $(\operatorname{coded} 1-3=0$ and $4-5=1) "$ \\
\hline Suicidal ideation & $\begin{array}{l}\text { "During the past } 12 \text { months, did you ever seriously } \\
\text { consider attempting suicide?" }\end{array}$ & "Yes, $\mathrm{No}^{\prime}$ \\
\hline Suicide attempt & $\begin{array}{l}\text { "During the past } 12 \text { months, how many times did } \\
\text { you actually attempt suicide?" }\end{array}$ & $\begin{array}{l}\text { " } 1=0 \text { times to } 5=6 \text { or more times }(\text { coded } 1=0 \text { and } \\
\qquad 2-5=1) "\end{array}$ \\
\hline Bullied & $\begin{array}{c}\text { "During the past } 30 \text { days, on how many days were } \\
\text { you bullied?" }\end{array}$ & " $1=0$ days to $7=$ All 30 days $(1-2=0$ and $3-7=1) "$ \\
\hline Physical fights & $\begin{array}{l}\text { "During the past } 12 \text { months, how many times were } \\
\text { you in a physical fight?" }\end{array}$ & $\begin{array}{c}" 1=0 \text { times to } 8=12 \text { or more times }(1-2=0 \\
\text { and } 3-8=1) "\end{array}$ \\
\hline Hunger & $\begin{array}{c}\text { "During the past } 30 \text { days, how often did you go } \\
\text { hungry because there was not enough food in your } \\
\text { home?" }\end{array}$ & $" 1=$ never to $5=$ always $(\operatorname{coded} 1-3=0$ and $4-5=1) "$ \\
\hline Parental tobacco use & $\begin{array}{l}\text { "Which of your parents or guardians use any form of } \\
\text { tobacco?" }\end{array}$ & $" 1=$ neither to $4=$ both $(\operatorname{coded} 1=0$ and $2-4=1) "$ \\
\hline Passive smoking & $\begin{array}{l}\text { "During the past } 7 \text { days, on how many days have } \\
\text { people smoked in your presence?" }\end{array}$ & $" 1=0$ days to $5=$ all 7 days $(\operatorname{coded} 1-4=0$ and $5=1) "$ \\
\hline School truancy & $\begin{array}{l}\text { "During the past } 30 \text { days, on how many days did you } \\
\text { miss classes or school without permission?" }\end{array}$ & $\begin{array}{l}\text { " } 1=0 \text { days to } 10 \text { or more days (coded } 1-2=0 \text { and } \\
\qquad 3-5=1) "\end{array}$ \\
\hline \multicolumn{3}{|l|}{ Health risk behaviors } \\
\hline Current tobacco use & $\begin{array}{l}\text { "During the past } 30 \text { days, on how many days did you } \\
\text { smoke cigarettes/use any tobacco products other than } \\
\text { cigarettes, such as country specific examples?" }\end{array}$ & $\begin{array}{c}\text { " } 1=0 \text { days to } 7=\text { All } 30 \text { days }(\text { coded } 1=0 \\
\text { and } 2-7=1) "\end{array}$ \\
\hline Current cannabis use & $\begin{array}{c}\text { "During the past } 30 \text { days, how many times have you } \\
\text { used marijuana?" }\end{array}$ & $\begin{array}{c}\text { "1 } 1=0 \text { times to } 5=20 \text { or more times }(\text { coded } 1=\text { and } \\
\qquad 2-5=1) "\end{array}$ \\
\hline Ever drunk & $\begin{array}{l}\text { "During your life, how many times did you drink so } \\
\text { much alcohol that you were really drunk?" }\end{array}$ & $\begin{array}{l}\text { " } 1=0 \text { times to } 4=10 \text { or more times }(\text { coded } 1=0 \text { and } \\
\qquad 2-4=1) "\end{array}$ \\
\hline Physical education & $\begin{array}{l}\text { "During this school year, on how many days did you } \\
\text { go to physical education (PE) class each week?" }\end{array}$ & $\begin{array}{l}\text { " } 1=0 \text { days to } 6=5 \text { or more days }(\text { coded } 1=1 \text { and } \\
\qquad 2-6=0) "\end{array}$ \\
\hline Injury & $\begin{array}{c}\text { "During the past } 12 \text { months, how many times were } \\
\text { you seriously injured?" }\end{array}$ & 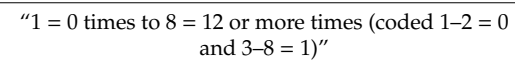 \\
\hline Soft drink intake & $\begin{array}{l}\text { "During the past } 30 \text { days, how many times per day } \\
\text { did you usually drink carbonated soft drinks, such as } \\
\text { country specific examples? (Do not include diet soft } \\
\text { drinks.)" }\end{array}$ & $\begin{array}{c}\text { "1 = never to }=5 \text { or more times a day }(\operatorname{coded} 1-3=0 \\
\text { and } 4-6=1) "\end{array}$ \\
\hline \multicolumn{3}{|l|}{ Protective factors } \\
\hline Peer support & $\begin{array}{l}\text { "During the past } 30 \text { days, how often were most of the } \\
\text { students in your school kind and helpful?" }\end{array}$ & $\begin{array}{c}\text { " } 1=\text { n ever to } 5=\text { always }(\operatorname{coded} 1-2=0, \\
3=2 \text { and } 4-5=1) "\end{array}$ \\
\hline Parental supervision & $\begin{array}{c}\text { "During the past } 30 \text { days, how often did your parents } \\
\text { or } \\
\text { guardians check to see if your homework was done?" }\end{array}$ & $" 1=$ never to $5=$ always $(\operatorname{coded} 1-3=0$ and $4-5=1) "$ \\
\hline Parental connectedness & $\begin{array}{c}\text { "During the past } 30 \text { days, how often did your parents } \\
\text { or } \\
\text { guardians understand your problems and worries?" }\end{array}$ & $" 1=$ never to $5=$ always $(\operatorname{coded} 1-3=0$ and $4-5=1) "$ \\
\hline Parental bonding & $\begin{array}{l}\text { "During the past } 30 \text { days, how often did your parents } \\
\text { or guardians really know what you were doing with } \\
\text { your free time?" }\end{array}$ & $" 1=$ never to $5=$ always $(\operatorname{coded} 1-3=0$ and $4-5=1) "$ \\
\hline Parental respect for privacy & $\begin{array}{c}\text { "During the past } 30 \text { days, how often did your parents } \\
\text { or guardians go through your things without your } \\
\text { approval?" }\end{array}$ & $" 1=$ never to $5=$ always $(\operatorname{coded} 1-3=0$ and $4-5=1) "$ \\
\hline
\end{tabular}




\subsection{Data Analysis}

Secondary data analyses were conducted using "STATA software version 15.0 (Stata Corporation, College Station, TX, USA)". Descriptive statistics were used to describe the sample. Logistic regression was used on the whole sample to identify the predictors of individual sexual risk behaviors (non-birth control use in the last sexual encounter, non-condom use in the last sexual encounter, multiple sexual partners, early sexual debut, and ever having had sex) and a summary measure of four sexual risk behaviors (having $\geq 2$ sexual risk behaviors). Co-variates were included based on previous literature reviews $[11-14,24]$. Taylor linearization procedures were utilized in all the statistical operations to account for the sampling weight and the multistage design of the study. Missing cases were not included in the analysis. The level of significance was set at $p<0.05$.

\section{Results}

\subsection{Characteristics of the Sample and Sexual Behavior}

The sample consisted of 9143 school adolescents from the Dominican Republic, Jamaica, Suriname, and Trinidad and Tobago; the overall mean age was 15.3 years $(S D=1.6)$. More than two in five students (41.4\%) had ever had sex, ranging from $26.4 \%$ in Trinidad and Tobago to $48.1 \%$ in Jamaica. Among those who had ever been sexually active, $58.6 \%$ had had an early sexual debut ( $\leq 14$ years), ranging from $47.9 \%$ in Trinidad and Tobago to $67.0 \%$ in Jamaica; $58.8 \%$ had had $\geq 2$ sexual partners, ranging from $43.3 \%$ in Trinidad and Tobago to $65.0 \%$ in Jamaica; $28.4 \%$ had used condoms in their last sexual encounter, ranging from $26.6 \%$ in the Dominican Republic to $33.8 \%$ in Trinidad and Tobago; $41.9 \%$ had used methods of birth control in their last sexual encounter, ranging from $36.0 \%$ in the Dominican Republic to $52.4 \%$ in Jamaica. Of the whole sample, $31.9 \%$ were involved in multiple $(\geq 2)$ sexual risk behaviors, ranging from $16.5 \%$ in Trinidad and Tobago to $40.3 \%$ in Jamaica. Additional characteristics of the sample are described in Table 3.

\subsection{Associations with Sexual Risk Behavior}

In an adjusted logistic regression analysis, male sex was associated with ever having had sex (AOR: 4.41, 95\% CI: 3.62-5.36), an early sexual debut (AOR: 7.26, 95\% CI: 5.89-9.62), multiple sexual partners (AOR: 7.95, 95\% CI: 5.39-11.84), non-condom use in the last sexual encounter (AOR: 2.89, 95\% CI: 1.91-4.40), non-birth control use in the last sexual encounter (AOR: 3.84, 95\% CI: 2.45-6.02), and multiple sexual risk behavior (AOR: 7.16, 95\% CI: 5.61-9.15). Compared to participants aged 15 years or less, the participants aged 16 years or older were more likely to have ever had sex (AOR: 2.75, 95\% CI: 1.66-4.55), to have had multiple sexual partners (AOR: 2.59, 95\% CI: 1.84-3.65), to have not used a condom in their last sexual encounter (AOR: 1.73, 95\% CI: 1.31-2.28), to have not used birth control in their last sexual encounter (AOR: 1.74, 95\% CI: 1.14-2.65), and to have engaged in multiple sexual risk behaviors (AOR: $2.89,95 \%$ CI: 1.68-4.98).

Regarding emotional-contextual factors, students with high psychological distress had increased odds of sexual initiation, non-condom use, multiple sexual partners, early sexual debut, and multiple sexual risk behaviors. Having been frequently involved in physical fights was associated with multiple sexual risk behaviors, sexual initiation, early sexual debut, non-birth control use, and multiple sexual partners. Parental tobacco use increased the odds for having ever had sex and non-birth control use, while school truancy was positively associated with ever having had sex and multiple sexual risk behaviors. Bullying victimization, hunger, and passive smoking were not significantly associated with any of the sexual risk behaviors. 
Table 3. Sample characteristics and sexual behavior types among adolescents in four Caribbean countries, 2016-2017.

\begin{tabular}{|c|c|c|c|c|c|c|c|}
\hline Study Variable & All & Ever Sex & $\begin{array}{c}\text { Early } \\
\text { Sexual } \\
\text { Debut }^{\text {a }}\end{array}$ & $\begin{array}{c}\text { Multiple } \\
\text { Sexual } \\
\text { Partners }\end{array}$ & $\begin{array}{c}\text { Non-Condom } \\
\text { Use }^{\mathrm{a}}\end{array}$ & $\begin{array}{l}\text { Non-Birth } \\
\text { Control } \\
\text { Use }^{\text {a }}\end{array}$ & $\begin{array}{l}\text { Multiple } \\
\text { Risk } \\
\text { Factors }\end{array}$ \\
\hline & $\mathrm{N}(\%)$ & $\mathrm{N}=2691$ & $\mathrm{~N}=1479$ & $\mathrm{~N}=1374$ & $\mathrm{~N}=794$ & $\mathrm{~N}=1074$ & $\mathrm{~N}=1888$ \\
\hline Sociodemographic variables & & $\%$ & $\%$ & $\%$ & $\%$ & $\%$ & $\%$ \\
\hline All & 9143 & 41.4 & 58.6 & 58.8 & 28.4 & 41.9 & 31.9 \\
\hline Country & & & & & & & \\
\hline Dominican Republic & $1481(16.2)$ & 41.8 & 55.9 & 58.0 & 26.6 & 36.0 & 31.7 \\
\hline Jamaica & $1667(18.2)$ & 48.1 & 67.9 & 65.0 & 31.5 & 52.4 & 40.3 \\
\hline Suriname & $2126(23.3)$ & 32.6 & 49.5 & 51.4 & 30.6 & 51.0 & 24.0 \\
\hline Trinidad and Tobago & $3869(42.3)$ & 26.4 & 47.9 & 43.3 & 33.8 & 45.1 & 16.5 \\
\hline \multicolumn{8}{|l|}{ Age in years } \\
\hline 13 or less & $2406(26.4)$ & 24.6 & 63.4 & 46.3 & 30.4 & 41.0 & 14.5 \\
\hline $14-15$ & 4017 (44.1) & 33.3 & 73.8 & 53.5 & 28.0 & 43.9 & 26.6 \\
\hline 16 or more & $2677(29.4)$ & 52.6 & 49.8 & 63.3 & 28.3 & 40.8 & 41.0 \\
\hline \multicolumn{8}{|l|}{ Sex } \\
\hline Female & $4816(50.7)$ & 26.8 & 36.1 & 37.5 & 28.8 & 35.9 & 16.2 \\
\hline Male & $4221(49.3)$ & 55.9 & 69.6 & 69.1 & 27.9 & 45.1 & 48.5 \\
\hline \multicolumn{8}{|l|}{ Emotional-contextual factors } \\
\hline \multicolumn{8}{|l|}{ Psychological distress } \\
\hline Low & $5028(62.3)$ & 36.6 & 60.4 & 60.9 & 24.8 & 42.9 & 28.8 \\
\hline Moderate & $1788(19.4)$ & 46.0 & 59.3 & 57.9 & 37.2 & 45.8 & 35.7 \\
\hline High & $1665(18.4)$ & 48.4 & 50.1 & 54.3 & 30.8 & 36.4 & 34.5 \\
\hline Bullied in past month (3-30 days) & $822(9.1)$ & 50.7 & 53.9 & 57.9 & 33.5 & 48.6 & 35.5 \\
\hline In physical fight in past year ( $\geq 2$ times) & $1399(12.9)$ & 62.8 & 75.4 & 63.2 & 29.8 & 51.0 & 54.4 \\
\hline Mostly/always feeling hungry & $701(4.6)$ & 50.5 & 60.9 & 64.4 & 35.4 & 38.5 & 40.8 \\
\hline Parental tobacco use & $2190(16.2)$ & 54.0 & 60.4 & 57.9 & 26.7 & 50.4 & 41.9 \\
\hline Passive smoking (all past 7 days) & $1733(16.7)$ & 52.7 & 65.7 & 68.6 & 33.6 & 50.0 & 44.4 \\
\hline School truancy (3-30 days) & $774(9.5)$ & 65.6 & 59.1 & 62.4 & 36.9 & 37.3 & 53.1 \\
\hline \multicolumn{8}{|l|}{ Health risk behaviors } \\
\hline Current tobacco use & $1222(13.8)$ & 72.5 & 74.5 & 70.8 & 29.1 & 41.5 & 63.6 \\
\hline Current cannabis use & $563(6.4)$ & 79.4 & 72.7 & 63.8 & 36.7 & 55.0 & 70.6 \\
\hline Ever drunk & $2148(28.6)$ & 63.5 & 64.5 & 66.5 & 32.3 & 45.9 & 54.1 \\
\hline No physical education & $2674(30.7)$ & 49.2 & 53.4 & 59.7 & 30.0 & 40.0 & 38.1 \\
\hline Injury in past 12 months ( $\geq 2$ times) & $1492(15.5)$ & 53.3 & 57.6 & 60.2 & 30.6 & 46.4 & 41.4 \\
\hline Soft drink intake ( $\geq 2$ drinks/day) & $3899(46.8)$ & 45.0 & 60.1 & 62.1 & 28.3 & 45.6 & 36.1 \\
\hline \multicolumn{8}{|l|}{ Protective factors } \\
\hline \multicolumn{8}{|l|}{ Peer support } \\
\hline Low & $3004(31.2)$ & 46.3 & 59.3 & 52.7 & 33.7 & 42.6 & 35.2 \\
\hline Moderate & $2701(29.3)$ & 37.1 & 60.6 & 59.5 & 30.6 & 47.1 & 28.4 \\
\hline High & $3052(39.6)$ & 39.2 & 56.8 & 63.1 & 23.6 & 39.2 & 31.2 \\
\hline \multicolumn{8}{|l|}{ Parental support } \\
\hline Low & $3554(38.9)$ & 48.2 & 61.3 & 58.5 & 33.4 & 46.9 & 37.8 \\
\hline Moderate & $2249(27.5)$ & 38.5 & 57.9 & 63.6 & 24.3 & 45.2 & 30.2 \\
\hline High & $2526(33.6)$ & 32.2 & 53.6 & 53.9 & 27.1 & 33.7 & 24.4 \\
\hline
\end{tabular}

In terms of health risk behaviors, current tobacco use was associated with an early sexual debut, multiple sexual partners, and multiple sexual risk behaviors, while current cannabis use and ever having been drunk were associated with four sexual risk behaviors. Frequent soft drink intake increased the odds of non-birth control use, multiple sexual partners, and multiple sexual risk behaviors. Nonattendance of physical education and frequent physical injury were not significantly associated with any of the sexual risk behaviors.

Regarding protective factors, high peer support increased the odds of multiple sexual partners, and moderate parental support was protective against non-condom use (see Tables 4 and 5). 
Table 4. Associations with sexual initiation, early sexual debut, and multiple sexual partners.

\begin{tabular}{|c|c|c|c|}
\hline Variable & Sexual Initiation & Early Sexual Debut & $\begin{array}{l}\text { Multiple Sexual } \\
\text { Partners }\end{array}$ \\
\hline & AOR $(95 \% \mathrm{CI})$ & AOR $(95 \% \mathrm{CI})$ & AOR $(95 \% \mathrm{CI})$ \\
\hline \multicolumn{4}{|l|}{ Sociodemographic factors } \\
\hline \multicolumn{4}{|l|}{ Country } \\
\hline Dominican Republic & 1 (Reference) & 1 (Reference) & 1 (Reference) \\
\hline Jamaica & $1.13(0.72,1.78)$ & $1.61(0.87,2.98)$ & $1.70(0.90,3.20)$ \\
\hline Suriname & $0.66(0.40,1.08)$ & $0.55(0.36,0.86)^{* *}$ & $0.54(0.27,1.09)$ \\
\hline Trinidad and Tobago & $0.47(0.29,0.75)^{* *}$ & $0.44(0.24,0.81)^{* *}$ & $0.39(0.19,0.80) *$ \\
\hline \multicolumn{4}{|l|}{ Age in years } \\
\hline 15 or less & 1 (Reference) & 1 (Reference) & 1 (Reference) \\
\hline 16 or more & $2.75(1.66,4.55)^{* * *}$ & $0.92(0.67,1.27)$ & $2.59(1.84,3.65)^{* * *}$ \\
\hline \multicolumn{4}{|l|}{ Sex } \\
\hline Female & 1 (Reference) & 1 (Reference) & 1 (Reference) \\
\hline Male & $4.41(3.62,5.36)^{* * *}$ & $7.26(5.89,9.62)^{* * *}$ & $7.95(5.39,11.84)^{* * *}$ \\
\hline \multicolumn{4}{|l|}{ Emotional-contextual factors } \\
\hline \multicolumn{4}{|l|}{ Psychological distress } \\
\hline Low & 1 (Reference) & 1 (Reference) & 1 (Reference) \\
\hline Moderate & $1.49(1.15,1.93)^{* *}$ & $1.45(1.07,1.96)$ * & $1.33(0.83,2.14)$ \\
\hline High & $1.56(1.12,2.16)^{* *}$ & $1.62(1.04,2.54) *$ & $1.61(1.13,2.30)^{* * *}$ \\
\hline Bullied in past month (3-30 days) & $0.97(0.67,1.42)$ & $0.86(0.51,1.54)$ & $0.75(0.44,1.25)$ \\
\hline $\begin{array}{c}\text { In physical fight in past year }(\geq 2 \\
\text { times) }\end{array}$ & $1.77(1.25,2.49)^{* * *}$ & $1.83(1.14,2.95)^{*}$ & $1.67(1.05,2.64)^{*}$ \\
\hline Mostly/always feeling hungry & $0.90(0.50,1.61)$ & $0.56(0.33,1.00)$ & $1.07(0.36,3.14)$ \\
\hline Parental tobacco use & $1.54(1.08,2.20)^{*}$ & $1.09(0.74,1.64)$ & $1.38(0.97,1.95)$ \\
\hline Passive smoking (all past 7 days) & $1.08(0.74,1.57)$ & $1.14(0.76,1.72)$ & $1.34(0.83,2.18)$ \\
\hline School truancy (3-30 days) & $2.31(1.58,3.36)^{* * *}$ & $1.59(0.84,3.01)$ & $1.61(0.86,3.01)$ \\
\hline \multicolumn{4}{|l|}{ Health risk behaviors } \\
\hline Current tobacco use & $1.28(0.84,1.96)$ & $2.27(1.42,3.61)^{* * *}$ & $2.14(1.37,3.34) * * *$ \\
\hline Current cannabis use & $2.66(1.61,4.40) * * *$ & $2.10(1.05,4.17) *$ & $1.20(0.60,2.45)$ \\
\hline Ever drunk & $2.65(1.90,3.70)^{* * *}$ & $2.63(1.88,3.68)^{* * *}$ & $2.66(1.98,3.57)^{* * *}$ \\
\hline No physical education & $1.23(0.89,1.70)$ & $1.12(0.71,1.74)$ & $1.40(0.91,2.15)$ \\
\hline $\begin{array}{c}\text { Injury in past } 12 \text { months }(\geq 2 \\
\text { times) }\end{array}$ & $1.14(0.79,1.63)$ & $0.82(0.56,1.19)$ & $1.06(0.75,1.48)$ \\
\hline Soft drink intake ( $\geq 2$ drinks/day) & $1.28(1.08,1.57)^{*}$ & $1.28(0.96,1.70)$ & $1.52(1.12,2.06) * *$ \\
\hline \multicolumn{4}{|l|}{ Protective factors } \\
\hline \multicolumn{4}{|l|}{ Peer support } \\
\hline Low & 1 (Reference) & 1 (Reference) & 1 (Reference) \\
\hline Moderate & $0.73(0.50,1.07)$ & $0.98(0.66,1.47)$ & $0.96(0.60,1.54)$ \\
\hline High & $0.82(0.58,1.16)$ & $1.02(0.70,1.46)$ & $1.45(1.08,1.95)$ * \\
\hline \multicolumn{4}{|l|}{ Parental support } \\
\hline Low & 1 (Reference) & 1 (Reference) & 1 (Reference) \\
\hline Moderate & $0.90(0.71,1.15)$ & $1.02(0.62,1.70)$ & $1.15(0.79,1.66)$ \\
\hline High & $1.01(0.75,1.36)$ & $1.00(0.69,1.45)$ & $1.12(0.77,1.65)$ \\
\hline
\end{tabular}

AOR $=$ Adjusted Odds Ratio; ${ }^{* * *} p<0.001 ;{ }^{* *} p<0.01{ }^{*} p<0.05$. 
Table 5. Associations with non-condom and non-birth control use and multiple sexual risk behaviors.

\begin{tabular}{|c|c|c|c|}
\hline Variable & Non-Condom Use & Non-Birth Control Use & $\begin{array}{c}\text { Multiple Sexual Risk } \\
\text { Behaviors }\end{array}$ \\
\hline & $\mathrm{AOR}(95 \% \mathrm{CI})$ & $\mathrm{AOR}(95 \% \mathrm{CI})$ & $\mathrm{AOR}(95 \% \mathrm{CI})$ \\
\hline \multicolumn{4}{|l|}{ Sociodemographic factors } \\
\hline \multicolumn{4}{|l|}{ Country } \\
\hline Dominican Republic & 1 (Reference) & 1 (Reference) & 1 (Reference) \\
\hline Jamaica & $1.05(0.61,1.80)$ & $1.40(0.76,2.56)$ & $1.54(0.93,2.57)$ \\
\hline Suriname & $0.84(0.48,1.49)$ & $0.68(0.42,1.10)$ & $0.68(0.41,1.13)$ \\
\hline Trinidad and Tobago & $0.82(0.46,1.44)$ & $0.63(0.34,1.16)$ & $0.44(0.24,0.79) * *$ \\
\hline \multicolumn{4}{|l|}{ Age in years } \\
\hline 15 or less & 1 (Reference) & 1 (Reference) & 1 (Reference) \\
\hline 16 or more & $1.73(1.31,2.28)^{* * *}$ & $1.74(1.14,2.65)^{*}$ & $2.89(1.68,4.98)^{* * *}$ \\
\hline \multicolumn{4}{|l|}{ Sex } \\
\hline Female & 1 (Reference) & 1 (Reference) & 1 (Reference) \\
\hline Male & $2.89(1.91,4.40)^{* * *}$ & $3.84(2.45,6.02) * * *$ & $7.16(5.61,9.15)^{* * *}$ \\
\hline \multicolumn{4}{|l|}{ Emotional-contextual factors } \\
\hline \multicolumn{4}{|l|}{ Psychological distress } \\
\hline Low & 1 (Reference) & 1 (Reference) & 1 (Reference) \\
\hline Moderate & $1.83(1.12,2.99)$ * & $1.18(0.86,1.63)$ & $1.55(1.10,2.18) *$ \\
\hline High & $2.05(1.25,3.35)^{* *}$ & $1.18(0.76,1.82)$ & $1.82(1.28,2.57)^{* * *}$ \\
\hline Bullied in past month (3-30 days) & $1.15(0.72,1.85)$ & $1.15(0.66,2.02)$ & $0.67(0.42,1.08)$ \\
\hline $\begin{array}{l}\text { In physical fight in past year } \\
\text { ( } \geq 2 \text { times) }\end{array}$ & $1.13(0.80,1.60)$ & $1.73(1.24,2.42)^{* *}$ & $1.90(1.22,2.95)^{* *}$ \\
\hline Mostly/always feeling hungry & $1.20(0.52,2.75)$ & $0.84(0.29,2.42)$ & $0.73(0.35,1.55)$ \\
\hline Parental tobacco use & $0.90(0.62,1.31)$ & $2.12(1.47,3.06) * * *$ & $1.18(0.89,1.56)$ \\
\hline Passive smoking (all past 7 days) & $1.45(0.98,2.16)$ & $1.18(0.66,2.11)$ & $1.06(0.73,1.52)$ \\
\hline School truancy (3-30 days) & $1.70(0.96,3.01)$ & $1.05(0.71,1.56)$ & $2.23(1.48,3.36) * * *$ \\
\hline \multicolumn{4}{|l|}{ Health risk behaviors } \\
\hline Current tobacco use & $1.00(0.83,1.58)$ & $0.87(0.53,1.44)$ & $1.64(1.05,2.56)^{*}$ \\
\hline Current cannabis use & $1.66(1.47,2.81)^{* * *}$ & $2.16(1.26,3.59)^{* *}$ & $2.57(1.51,4.38)^{* * *}$ \\
\hline Ever drunk & $2.03(0.79,1.81)$ & $1.93(1.28,2.90)^{* *}$ & $3.20(2.42,4.23) * * *$ \\
\hline No physical education & $1.20(0.71,1.61)$ & $1.19(0.76,1.85)$ & $1.19(0.79,1.78)$ \\
\hline $\begin{array}{l}\text { Injury in past } 12 \text { months } \\
\text { ( } \geq 2 \text { times) }\end{array}$ & $1.34(0.72,2.51)$ & $1.28(0.88,1.85)$ & $1.07(0.67,1.69)$ \\
\hline Soft drink intake ( $\geq 2$ drinks/day) & $1.07(0.71,1.61)$ & $1.58(1.07,2.32)^{*}$ & $1.47(1.09,1.98)^{*}$ \\
\hline \multicolumn{4}{|l|}{ Protective factors } \\
\hline \multicolumn{4}{|l|}{ Peer support } \\
\hline Low & 1 (Reference) & 1 (Reference) & 1 (Reference) \\
\hline Moderate & $0.68(0.42,1.09)$ & $0.96(0.64,1.45)$ & $0.79(0.51,1.21)$ \\
\hline High & $0.68(0.40,1.17)$ & $1.03(0.70,1.53)$ & $0.97(0.67,1.42)$ \\
\hline \multicolumn{4}{|l|}{ Parental support } \\
\hline Low & 1 (Reference) & 1 (Reference) & 1 (Reference) \\
\hline Moderate & $0.61(0.42,0.89)^{* *}$ & $0.84(0.61,1.15)$ & $1.13(0.80,1.60)$ \\
\hline High & $1.17(0.61,2.25)$ & $0.74(0.50,1.10)$ & $1.25(0.86,1.81)$ \\
\hline
\end{tabular}

AOR = Adjusted Odds Ratio; ${ }^{* * *} p<0.001,{ }^{* *} p<0.01,{ }^{*} p<0.05$.

\section{Discussion}

The results show a high proportion of sexual initiation (41.4\%) among school-going adolescents in four Caribbean countries, which seems higher than the 2013 estimates for the Caribbean $(20 \%$ among girls and $40 \%$ among boys) [2], in a community survey among adolescents in seven African countries (25.9\%) [25]; among school adolescents in 30 countries in Europe, Israel, and Canada (27\%) [26]; and in 
a 10 European countries study among adolescents (18.8\%) [27]. There was some country variation in the prevalence of sexual initiation, with Jamaica being the highest (48.1\%) and Trinidad and Tobago being the lowest (26.4\%). Two previous local studies among adolescents in Jamaica also reported a high proportion of sexual initiation (32\% among girls and 54\% among boys) [6], and in Kingston and St Andrew (58.6\%) [7].

Among sexually active students, $28.4 \%$ had not used condoms in their last sexual encounter, which is a significant improvement compared to 2013 or earlier data among adolescents in the Caribbean (38\%) [2], Haiti (42.3\%) [3], seven African countries (46\% among girls and 49\% among boys) [25], and four countries in Southeast Asia (46.9\%) [24], but similar to adolescents in Uganda (22.7\%) [28]. Moreover, high sexual risk behavior was found in terms of early sexual debut ( $\leq 15$ years) $(58.8 \%)$, multiple sex partners (58.8\%), non-birth control use (last sex) $(41.9 \%)$, and engagement in two or more sexual risk behaviors (31.9\%). The proportion of early sexual debut among adolescents in the four Caribbean countries was higher in this study (58.8\%) than in a previous study in the Caribbean in 2007-2009 (26.9\%) [5] and in seven African countries ( $<15$ years, 21\% among girls and $28 \%$ among boys) [18], but similar to 2013 or earlier Caribbean research data (56\% of girls and $79 \%$ of boys) [2]. The proportion of multiple sexual partners (58.8\%) among adolescents in the Caribbean in this study was higher than in 2013 research in the Caribbean (31\% among boys and 10\% among girls) [2], in Kingston and St Andrew in Jamaica (31.8\%) [7], in another study in Jamaica (12\% among girls and $52 \%$ among boys) [6], in Ghana (32.5\%) [29], and in 15 year-olds in 10 European countries (52.4\%) [27], but lower compared to a local study of 200 adolescents in Haiti (62.2\%) [3].

The proportion of non-contraceptive use (41.9\%) among adolescents in the Caribbean in this study was higher than in Europe, Israel, and Canada (14\%) [26]. Although the prevalence of birth control use $(64 \%)$ and condom use $(73.4 \%)$ in the last sexual encounter among adolescents in Dominican Republic in this study was the highest among the four Caribbean study countries, the adolescent fertility rate was the highest in the Caribbean (108.7 per 1000 adolescent women) [2]. This disparity may be related to our sample selection of middle school students rather than out-of-school adolescents. Most sexual risk behaviors were the highest in Jamaica and the lowest in Trinidad and Tobago, which concurs with some previous research [6,7]. Overall, it appears that protected sexual intercourse increased but also early sexual debut and multiple sexual partners increased among adolescents in the four Caribbean countries. It is possible that increased HIV prevention campaigns led to a high proportion of condom use among the studied adolescents.

Male sex and/or older adolescents were associated with almost all individual sexual risk behaviors (sexual initiation, multiple sexual partners, early sexual debut, and unprotected sex) and multiple sexual risk behaviors. Similar results were shown in previous investigations $[3,10,24,29,30]$, which may support the case of sexual risk intervention programs targeting male adolescents at an earlier age than their female counterparts. Unlike in former research [31], this study showed a non-association between frequent hunger experiences (or lower socioeconomic status) and sexual risk behaviors. A possible explanation for this finding is that the prevalence of hunger was low in this study and the concept of socioeconomic status was assessed more comprehensively in other studies, such as including the education of the household head and a list of household possessions [31]. Frequent involvement in physical fights increased the odds for all individual sexual risk behaviors (except for non-condom use) and multiple sexual behaviors. These findings are in line with those of a previous multi-country study among school adolescents [12]. In addition, frequent soft drink consumption was positively associated with non-birth control use, multiple sexual risk behaviors, and multiple sexual partners in this study. In previous research, soft drink intake has been found o be associated with various health risk behaviors, including having had multiple sexual partners and unprotected sex [13,32]. Further research is needed to investigate this novel finding in the Caribbean.

In agreement with a number of previous studies $[3,9,29,30,33]$, this survey showed that substance use (tobacco, cannabis, and alcohol) increased the likelihood of engaging in most individual as well as multiple sexual risk behaviors. In line with previous research [10,29,34-36], psychological distress 
was in this study associated with most single and multiple sexual risk behaviors. The findings from this research seem to show a clustering between psychological distress (an internalizing factor), substance use, interpersonal violence such as physical fighting (an externalizing factor), and sexual risk behavior. This finding may have implications for integrating psychological distress, substance use, and interpersonal violence in sexual and reproductive health promotion programs.

The study showed that attending school was protective against sexual initiation and having multiple sexual risk behaviors, which is consistent with previous research findings [10,30]. Interventions preventing school truancy and promoting school attendance may also be beneficial in sexual risk behavior reduction. Unlike some former research studies $[3,9,30,37,38]$, this survey showed that peer and parental support was not protective against sexual risk behavior. In fact, high peer support increased the odds of having multiple sexual partners. It is possible that this behavior (having multiple sexual partners) is supported by peers and consequently increases it. In a study among school adolescents in the Bahamas, "greater perceived peer risk involvement predicted higher sexual risk behavior index scores" [38]. This could mean that sexual health promotion should include peer norms and perceptions. Comprehensive and timely sexual and reproductive health education is needed in secondary schools in the Caribbean [39].

\section{Limitations of the Study}

The GSHS only includes adolescents that attend school, excluding out-of-school youth. Adolescents who have dropped out of school may be more vulnerable to sexual risk behavior. The GSHS was cross-sectional by design, which precludes causative inference between the study variables. Furthermore, the self-reported data collection may have led to biased responses, in particular regarding sensitive issues such as sexual behavior. The GSHS does not provide a definition of "sexual intercourse", and therefore it is possible that some students misinterpreted the meaning, but the same question is used in various other surveys among adolescents (e.g., [27]).

\section{Conclusions}

More than two in five students had ever had sex among school-going adolescents in four Caribbean countries. Among the sexually active participants, two in five had had non-birth control use, almost three in five had had an early sexual debut and multiple sexual partners, and almost three in ten had not used condoms in their last sexual encounter. Sexual risk behaviors were higher in students who had psychological distress, engaged in substance use, had frequent soft drink intake, were older, were male, and were absent from school. Taking the identified factors associated with sexual risk behaviors into account will be important in the design and scaling up of sexuality and reproductive health education among school adolescents in the Dominican Republic, Jamaica, Suriname, and Trinidad and Tobago.

\section{Availability of Data and Materials}

The data for the current study are publicly available at the World Health Organization NCD Microdata Repository (URL: https://extranet.who.int/ncdsmicrodata/index.php/catalog).

Author Contributions: All the authors fulfill the criteria for authorship. S.P. and K.P. conceived and designed the research, performed the statistical analysis, drafted the manuscript, and made critical revision of the manuscript for key intellectual content. All authors have read and agreed to the published version of the manuscript.

Funding: This research received no external funding.

Acknowledgments: The data source, the World Health Organization NCD Microdata Repository (URL: https://extranet.who.int/ncdsmicrodata/index.php/catalog), is hereby acknowledged.

Conflicts of Interest: The authors declare no conflict of interest. 


\section{References}

1. Bearinger, L.H.; Sieving, R.E.; Ferguson, J.; Sharma, V. Global perspectives on the sexual and reproductive health of adolescents: Patterns, prevention, and potential. Lancet 2007, 369, 1220-1231. [CrossRef]

2. Pan American Health Organization/World Health Organization. Situation Analysis of Adolescent Sexual and Reproductive Health and HIV in the Caribbean. 2013. Available online: https://www.paho.org/hq/ dmdocuments/2013/PAHO-ASRH-Executive-Summary2013.pdf (accessed on 10 September 2020).

3. Carver, J.W.; Dévieux, J.G.; Gaston, S.C.; Altice, F.L.; Niccolai, L.M. Sexual risk behaviors among adolescents in Port-au-Prince, Haiti. AIDS Behav. 2014, 18, 1595-1603. [CrossRef] [PubMed]

4. Pan Caribbean Partnership against HIV/AIDS. UNAIDS Report on the AIDS Epidemic Shows that 2020 Targets Will not be Met in the Caribbean. 2020. Available online: https:/pancap.org/pancap-releases/unaidsreport-on-the-aids-epidemic-shows-that-2020-targets-will-not-be-met-in-the-caribbean/ (accessed on 10 September 2020).

5. Peltzer, K.; Pengpid, S. Early Sexual Debut and Associated Factors among In-school Adolescents in Six Caribbean Countries. West Indian Med. J. 2015, 64, 351-356. [CrossRef] [PubMed]

6. Ishida, K.; Stupp, P.; McDonald, O. Prevalence and correlates of sexual risk behaviors among Jamaican adolescents. Int. Perspect. Sex Reprod. Health 2011, 37, 6-15. [CrossRef] [PubMed]

7. Longman-Mills, S.; Carpenter, K. Interpersonal competence and sex risk behaviours among Jamaican adolescents. West Indian Med. J. 2013, 62, 423-426. [CrossRef] [PubMed]

8. Jarrett, S.B.; Udell, W.; Sutherland, S.; McFarland, W.; Scott, M.; Skyers, N. Age at Sexual Initiation and Sexual and Health Risk Behaviors Among Jamaican Adolescents and Young Adults. AIDS Behav. 2018, 22 (Suppl. 1), 57-64. [CrossRef]

9. Pilgrim, N.A.; Blum, R.W. Protective and risk factors associated with adolescent sexual and reproductive health in the English-speaking Caribbean: A literature review. J. Adolesc. Health. 2012, 50, 5-23. [CrossRef]

10. Peltzer, K.; Pengpid, S. Risk and protective factors affecting sexual risk behaviour among school-aged adolescents in Fiji, Kiribati, Samoa, and Vanuatu. Asia Pac. J. Public Health 2016, 28, 404-415. [CrossRef] [PubMed]

11. Smith, L.; Grabovac, I.; Jacob, L.; López-Sánchez, G.F.; Yang, L.; Shin, J.I.; Sohn, M.; Ward, P.B.; McDermott, D.T.; Koyanagi, A. Bullying victimization and sexual behavior among adolescents aged 12-15 years from 53 countries: A global perspective. J. Sex Med. 2020. [CrossRef] [PubMed]

12. Smith, L.; Jacob, L.; López-Sánchez, G.F.; Grabovac, I.; Yang, L.; Pizzol, D.; Sigman, A.; McDermott, D.; Koyanagi, A. A Multicountry Study of the Violence-Related Risk Factors for Early Sexual Debut and Risky Sexual Behavior in Adolescents. J. Interpers. Violence 2020. [CrossRef]

13. Ziegler, A.M.; Temple, J.L. Soda Consumption is Associated with Risk-Taking Behaviors in Adolescents. Am. J. Health Behav. 2015, 39, 761-771. [CrossRef] [PubMed]

14. Yang, X.H.; Yuan, S.; Zhang, R.; Yu, J.F.; Nzala, S.H.; Wang, P.G.; He, Q.Q. Risky Sexual Behaviors and Associated Factors Among College Students in Lusaka, Zambia. Arch Sex Behav. 2019, 48, 2117-2123. [CrossRef] [PubMed]

15. UNAIDS. Terminology Guidelines. 2015. Available online: https://www.unaids.org/sites/default/files/media_ asset/2015_terminology_guidelines_en.pdf (accessed on 6 October 2020).

16. World Health Organization (WHO). Global School-Based Student Health Survey (GSHS). 2020. Available online: https:/www.who.int/ncds/surveillance/gshs/en/ (accessed on 10 September 2020).

17. World Factbook. Dominican Republic. 2020. Available online: https://www.cia.gov/library/PUBLICATIONS/ the-world-factbook/geos/dr.html (accessed on 6 October 2020).

18. World Factbook. Jamaica. 2020. Available online: https://www.cia.gov/library/publications/resources/theworld-factbook/geos/jm.html (accessed on 6 October 2020).

19. World Factbook. Suriname. 2020. Available online: https://www.cia.gov/library/publications/the-worldfactbook/geos/ns.html (accessed on 6 October 2020).

20. World Factbook. Trinidad and Tobago. 2020. Available online: https://www.cia.gov/library/publications/ resources/the-world-factbook/geos/td.html (accessed on 6 October 2020). 
21. UNESCO Institute for Statistics. School Enrollment, Secondary. 2020. Available online: uis.unesco.org (accessed on 6 October 2020).

22. Brener, N.D.; Collins, J.L.; Kann, L.; Warren, C.W.; Williams, B.I. Reliability of the Youth Risk Behavior Survey Questionnaire. Am. J. Epidemiol. 1995, 141, 575-580. [CrossRef]

23. Becker, A.E.; Roberts, A.L.; Perloe, A.; Bainivualiku, A.; Richards, L.K.; Gilman, S.E.; Striegel-Moore, R.H. Youth health-risk behavior assessment in Fiji: The reliability of Global School-based Student Health Survey content adapted for ethnic Fijian girls. Ethn. Health 2010, 15, 181-197. [CrossRef]

24. Peltzer, K.; Pengpid, S. Sexual risk behaviour and its correlates among adolescents in Indonesia, Laos, Thailand, and Timor-Leste: Results from national school surveys in 2015. Int. J. Adolesc. Med. Health 2020. [CrossRef] [PubMed]

25. Berhane, Y.; Canavan, C.R.; Darling, A.M.; Sudfeld, C.R.; Vuai, S.; Adanu, R.; Bärnighausen, T.; Dessie, Y.; Bukenya, J.N.; Guwatudde, D.; et al. The age of opportunity: Prevalence of key risk factors among adolescents 10-19 years of age in nine communities in sub-Saharan Africa. Trop. Med. Int. Health. 2020, 25, 15-32. [CrossRef]

26. Nic Gabhainn, S.; Baban, A.; Boyce, W.; Godeau, E. HBSC Sexual Health Focus Group. How well protected are sexually active 15-year olds? Cross-national patterns in condom and contraceptive pill use 2002-2006. Int. J. Public Health 2009, 54 (Suppl. 2), 209-215. [CrossRef]

27. Gambadauro, P.; Carli, V.; Wasserman, C.; Hadlaczky, G.; Sarchiapone, M.; Apter, A.; Balazs, J.; Bobes, J.; Brunner, R.; Cosman, D.; et al. Psychopathology is associated with reproductive health risk in European adolescents. Reprod. Health 2018, 15, 186. [CrossRef]

28. Twa-Twa, J.M.; Oketcho, S.; Siziya, S.; Muula, A.S. Prevalence and correlates of condom use at last sexual intercourse among in-school adolescents in urban areas of Uganda. East Afr. J. Public Health 2008, 5, $22-25$. [CrossRef]

29. Kugbey, N.; Ayanore, M.A.; Amu, H.; Asante, K.O.; Adam, A. International note: Analysis of risk and protective factors for risky sexual behaviours among school-aged adolescents. J. Adolesc. 2018, 68, 66-69. [CrossRef]

30. Mmari, K.; Blum, R.W. Risk and protective factors that affect adolescent reproductive health in developing countries: A structured literature review. Glob. Public Health 2009, 4, 350-366. [CrossRef]

31. Sanchez, Z.M.; Nappo, S.A.; Cruz, J.I.; Carlini, E.A.; Carlini, C.M.; Martins, S.S. Sexual behavior among high school students in Brazil: Alcohol consumption and legal and illegal drug use associated with unprotected sex. Clinics 2013, 68, 489-494. [CrossRef]

32. Pengpid, S.; Peltzer, K. High Carbonated Soft Drink Intake is Associated with Health Risk Behavior and Poor Mental Health among School-Going Adolescents in Six Southeast Asian Countries. Int. J. Environ. Res. Public Health 2019, 17, 132. [CrossRef] [PubMed]

33. Smith, L.; Jackson, S.E.; Jacob, L.; Grabovac, I.; Nisbet, L.A.; López-Sánchez, G.F.; McDermott, D.; Salmeron, A.G.; Yang, L.; Koyanagi, A. Association between cannabis use and sexual behavior among adolescents aged 12-15 years in 21 low- and middle-income countries. Addict Behav. 2019, 99, 106073. [CrossRef] [PubMed]

34. Page, R.M.; Hall, C.P. Psychosocial distress and alcohol use as factors in adolescent sexual behavior among sub-Saharan African adolescents. J. Sch. Health. 2009, 79, 369-379. [CrossRef] [PubMed]

35. Gambadauro, P.; Carli, V.; Hadlaczky, G.; Sarchiapone, M.; Apter, A.; Balazs, J.; Banzer, R.; Bobes, J.; Brunner, R.; Cosman, D.; et al. Correlates of sexual initiation among European adolescents. PLoS ONE 2018, 13, e0191451. [CrossRef] [PubMed]

36. Peltzer, K. Early sexual debut and associated factors among in-school adolescents in eight African countries. Acta Paediatr. 2010, 99, 1242-1247. [CrossRef] [PubMed]

37. Peltzer, K.; Pengpid, S. Prevalence and social correlates of sexual intercourse among school-going adolescents in Thailand. Sci. World J. 2011, 11, 1812-1820. [CrossRef] 
38. Wang, B.; Stanton, B.; Deveaux, L.; Li, X.; Lunn, S. Dynamic Relationships Between Parental Monitoring, Peer Risk Involvement and Sexual Risk Behavior Among Bahamian Mid-Adolescents. Int. Perspect. Sex Reprod. Health 2015, 41, 89-98. [CrossRef]

39. Dongarwar, D.; Salihu, H.M. Influence of Sexual and Reproductive Health Literacy on Single and Recurrent Adolescent Pregnancy in Latin America. J. Pediatr. Adolesc. Gynecol. 2019, 32, 506-513. [CrossRef]

Publisher's Note: MDPI stays neutral with regard to jurisdictional claims in published maps and institutional affiliations.

(C) 2020 by the authors. Licensee MDPI, Basel, Switzerland. This article is an open access article distributed under the terms and conditions of the Creative Commons Attribution (CC BY) license (http://creativecommons.org/licenses/by/4.0/). 